\title{
EINIGE BEMERKUNGEN ZUM INFOGRAFISCHEN IN DER VISUELLEN PUBLIZISTIK
}

\begin{abstract}
A few remarks concerning infographics in visual journalism
Infographics has become one the most popular forms used in contemporary communication. It is employed both in traditional media as well as electronic ones in order to present relations between various phenomena or explain particular mechanisms of action in a picturesque way. Infographics is a hybrid form which reaches out for disparate visual elements: linguistic, pictorial and diagram ones, and combines them in a nonlinear way. In media communication infographics occur both as an independent message, or as e.g. an element accompanying a press article and complementing it. The purpose of the article is to demonstrate that infographics as a visual plane can serve the economisation and rationalisation of media communication.
\end{abstract}

KEYWORDS: infographic, visual plane, multimodal texts

In der Zeit der visuellen Kommunikation, in der fast ausschließlich multimodal kommuniziert wird, erreicht die Welle des Infografischen fast alle Bereiche des sprachlichen Handelns. Obwohl die Infografik ihre Wurzeln im Informationsdesign hat, findet sie ihre größte Verbreitung im Journalismus. Das liegt u. a. an ihrer ergonomisch wirkenden Hybridität, die sich als multimodales Zusammenspiel aus Sprache, Bild und Zahl beschreiben lässt und die Infografiken zum spannenden Forschungsfeld für Disziplinen wie z. B. die Medienwissenschaft, Psychologie, Informatik, Kartografie oder Kulturgeschichte macht. Die interdisziplinäre Analyse von Infografiken ist von Vorteil, weil jede Disziplin ihre eigenen Akzente setzt, indem sie Infografiken aus einer anderen Sicht erforscht und beschreibt. Immer häufiger wird die Frage des Infografischen auch im Kontext der linguistischen Forschung thematisiert, weil Infografiken als Kommunikationsmittel nicht nur als Begleitmaterial eingesetzt werden, sondern auch separat erscheinen und als selbständige Textsorte betrachtet werden können (vgl. Stöckl, 2012). Infografiken verkörpern das, was in der medienlinguistischen Forschung mit der Hybridisierung der Kommunikation assoziiert wird. Ihre modulare Struktur, in

Magdalena Makowska - Uniwersytet Warmińsko-Mazurski, Olsztyn, magdalena.makowska@ uwm.edu.pl 
der sprachliche, bildliche und diagrammatische Ressourcen funktional und inhaltlich zusammenhängen und sich wechselseitig kontextualisieren, stellt eine Herausforderung sowohl für Produzenten als auch für Rezipienten dar, von denen eine bestimmte multimodale Kompetenz im Umgang mit solchen Botschaften erwartet wird. Deshalb ist die Infografik ein interessanter Gegenstand sowohl der medienlinguistischen, als auch der interdisziplinären Forschung.

Der vorliegende Beitrag setzt sich zum Ziel, Infografiken als Textsorte zu beschreiben und im Kontext der durch Hybridisierungsprozesse geprägten visuellen Kommunikation zu thematisieren. Als Datenbasis gelten Infografiken, die in deutschen Titeln wie z. B. dem Fokus und dem Stern im Zeitraum von Januar bis November 2015 erschienen sind. Gerade die Infografiken, die als journalistische Darstellungsform in den letzten Jahren eine enorme Dynamik erfahren, veranschaulichen, welchen Veränderungen die Medienlandschaft infolge von Hybridisierungsprozessen unterliegt. Die das Korpus bildenden Infografiken werden als multimodale Botschaften betrachtet, die zwischen den Disziplinen (Linguistik, Statistik, Informatik, Design, Medienwissenschaft), zwischen den Zeichensystemen (Bild, Sprache, Zahl), zwischen den verschiedenen Modi (geschriebene Sprache, Bild, Layout, Diagramm) und zwischen Linearität und Non-Linearität angesiedelt sind. Ihr besonderer Vorteil besteht darin, dass sie mit fast jedem Thema kommunizierbar sind: Infografisch lassen sich sowohl politische Wahlen als auch komplizierte Technologien darstellen. Infografiken gelten vor allem als Begleitmaterial von journalistischen oder wissenschaftlichen Texten. Immer häufiger werden sie aber auch als separate Sehflächen (Schmitz, 2011b) gebraucht, die keinen Text mehr begleiten, sondern diesen ersetzen, um einen Sachverhalt verständlich und anschaulich zu erklären. Gerade den separat gebrauchten Infografiken wird im vorliegenden Beitrag besondere Aufmerksamkeit geschenkt. Sie werden als komplexe, multimodale Superzeichen betrachtet, in denen diagrammatische, sprachliche und bildliche Ressourcen integriert werden und miteinander interagieren.

\section{Infografische Botschaften in der Medienlandschaft}

Die Geschichte der Infografik beginnt in den 1970er Jahren (vgl. Berger, 1989, S. 164; zit. nach Knieper, 1995, S. 3). Der Begriff, der ein Kompositum aus Information und Grafik ist und die sich permanent im Wandel befindliche Darstellungsform beschreibt, weist darauf hin, was sich als eigentliches Ziel des Infografischen verstehen lässt: Infografiken visualisieren Informationen. Der Begriff Information stammt vom lateinischen Wort informatio ab und bedeutet Bildung, Belehrung, Unterricht. Informatio lässt sich von informare (bilden, gestalten, unterrichten) ableiten und dieses Wort setzt sich wiederum aus in-formare zusammen (vgl. Hermann, 1982; zit. nach Gudehus, 2010, S. 2450). Der zweite Bestandteil des Kompositums, das Wort Grafik, stammt vom griechischen gráphein (schreiben, zeichnen, einritzen) ab (vgl. Gudehus, 2010, 
S. 866) und gilt auch als „Sammelbezeichnung für alle zeichnerischen Künste, die im Gegensatz zur Malerei von der Linie und nicht von der Farbe bestimmt sind“. Aus den Einzelbedeutungen des Kompositums ergibt sich, dass Infografiken als grafisch aufbereitete und präsentierte Informationen bezeichnet werden können (vgl. Radtke \& Pissani, 2001; zit. nach Gudehus, 2010, S. 2450). Während sich die ersten Infografiken auf statisch gebrauchte diagrammatische und sprachliche Ressourcen begrenzten, werden heutzutage immer häufiger interaktive Infografiken gebraucht, die von dynamischen Bildern Gebrauch machen und die dadurch die Interaktion zwischen Produzenten und Rezipienten ermöglichen. Weil sich der vorliegende Beitrag ausschließlich mit statischen, in Printmedien präsentierten Infografiken beschäftigt, wird denjenigen Definitionen gefolgt, in deren Zentrum die Darstellungselemente von Infografiken stehen und nicht ihre interaktionelle Funktion. Norbert Küpper, der darauf hinweist, dass Menschen von Natur aus visuell geprägt sind, definiert Infografiken als den dritten und schnellsten Weg der Informationsübermittlung neben Text und Bild (vgl. Küpper, 2010, S. 2). Somit wird hervorgehoben, dass zu den wichtigsten Aufgaben von Infografiken die Informationsvermittlung gehört, die aufgrund der Wahrnehmung erfolgt. Die Art und Weise, wie Inhalte visualisiert werden, beeinflusst, wie man sie wahrnimmt und ob es dem Rezipienten gelingt, alle Zusammenhänge, die durch die infografischen Ressourcen entstehen, richtig zu dekodieren. Die Rolle der Darstellungsform betonen auch Joachim Blum und Hans-Jürgen Bucher, die darauf verweisen, dass die Infografik affektives und kognitives Aufnehmen von Information, Bild und Textrezeption, Sehen und Lesen verbindet: ,[...] Erst die veranschaulichende Transferleistung macht die Informationsgrafik aus; sie ist eine Kombination aus Illustration und Text $[\ldots]^{\text {“ }}$ (Blum \& Bucher, 1998, S. 57). Während grafische Bestandteile der Infografik vor allem die Aufmerksamkeit des Rezipienten wecken und visuelle Informationen vermitteln sollen, werden mittels typografischer Elemente Zusammenhänge, Funktionen oder zeitliche Abläufe veranschaulicht (vgl. Bouchon, 2007, S. 16). Konstitutiv für Infografiken scheint die visuell aufbereitete Informationsvermittlung zu sein, die mittels diagrammatischer, sprachlicher und bildlicher Ressourcen erfolgt. Dieser Definition der Infografik wird im vorliegenden Beitrag gefolgt.

Die moderne Medienlandschaft ist durch die allgemeine Zunahme, Pluralisierung und Polarisierung der Berichterstattung gekennzeichnet. Hartmut Stöckl hält den multimodalen Text nicht für einen überkomplexen Sonderfall, sondern für den Normalfall des Kommunizierens, und weist darauf hin, dass monomodale Texte sprachwissenschaftlich projizierte Fiktionen sind (vgl. Stöckl, 2010, S. 47). Infolge der Entwicklung von neuen Technologien gibt es immer mehr Möglichkeiten, den Rezipienten schnell zu erreichen und ihn zielgerichtet anzusprechen. Dank den Neuen Medien unterliegt die Medienlandschaft auch einem formalen Wandel: Es entstehen immer neue Textsorten, die mediale Repertoires erweitern, neue Bezüge herstellen und Beziehungen zwischen Produzenten und Rezipienten so organisieren, dass immer mehr auf Interaktion gesetzt wird. Die Veränderungen, denen die Medienlandschaft unterliegt, lassen sich mittels 
zweier Prozesse beschreiben. Der eine heißt Differenzierung und bezieht sich auf mögliche Textsortenbezüge in größeren Verbünden. Der andere ist Hybridisierung und betrifft alle Veränderungen, denen eine Textsorte unterliegt oder die zur Entstehung neuer Textsorten führen können. Hartmut Stöckl weist darauf hin, dass „,beide Phänomene eng [zusammenhängen]: Ausdifferenzierungsprozesse können (müssen aber nicht) zu hybriden Textsorten führen; hybride Textsorten wiederum sind in der Lage, die Ausdifferenzierung einer Kommunikationsform (Zeitung) wesentlich zu unterstützen“ (Stöckl, 2015, S. 236). Die Neigung zur Hybridisierung und Ausdifferenzierung lässt sich in fast allen Bereichen der Kommunikation nachweisen und führt zur Entstehung neuer Möglichkeiten und Bedingungen der Verflechtung unterschiedlicher Ressourcen. Als Resultat dessen gelten neuartige Lösungen im Bereich der Textsorten. Infografiken scheinen zu exemplifizieren, wie sich eine Textsorte in der Zeit des medialen Wandels verändern kann. Sie sind durch ein gewisses $\mathrm{Maß}$ an funktionaler Stabilität gekennzeichnet, weil sie immer noch als der Informationsvermittlung dienende Botschaften identifiziert und interpretiert werden. Ihre strukturelle Stabilität ist allerdings begrenzt. Einerseits verfügen Infografiken über diagrammatische und sprachliche Elemente, die prototypisch für diese Gattung sind. Andererseits weisen sie aber auch eine starke Neigung zur strukturellen Flexibilität auf, die dadurch zum Ausdruck kommt, dass ihre grafische Form immer differenzierter wird. Im Kontext der Hybridisierung weisen Stefan Hauser und Martin Luginbühl darauf hin, dass

$[\ldots]$ die Form [...] einen analytischen Zugang zu materialisierten symbolischen Sinnwelten und somit einen analytischen Zugang zu kulturellen Werten [bietet], die immer über symbolische Formen etabliert und kommuniziert werden (müssen). (Hauser \& Luginbühl, 2015, S. 15)

Infografiken gelten nicht mehr als Textsorten, die durch eine Verschmelzung ausschließlich diagrammatischer und sprachlicher Elemente geprägt sind und die meistens als Begleitmaterial zu anderen Beiträgen fungieren. Die moderne Infografik ähnelt immer mehr einer hybriden Darstellungsform, die über eine vielschichtige, modulare Struktur verfügt und als solche mehrere kommunikative Funktionen übernehmen kann. Somit wird die Infografik als eine selbständige Textsorte betrachtet, die immer häufiger keinen Text mehr begleitet, sondern diesen ersetzt, seine Funktionen übernimmt und als separate Botschaft erscheint.

\section{Infografik als Sehfläche}

Kaum eine andere Darstellungsform erhebt die Zeichensysteme Bild, Sprache und Zahl derart zu ebenbürtigen Partnern wie die Infografik. Bildliche, sprachliche und diagrammatische Module verschränken sich in der Infografik zu einem emergenten Ganzen. Im Falle von denjenigen Botschaften, die als Phänomene der medialen 
Verbindung von sprachlichen, bildlichen und diagrammatischen Elementen gelten, spricht Thomas Lischeid über sog. „,diskontinuierliche Darstellungsformen“ (Lischeid, 2012, S. 8). Infografiken lassen sich als Darstellungsformen klassifizieren, weil sie auf medialer Ebene als Kombination verbaler und visueller Zeichenelemente und auf modaler Ebene als Kombination textueller (Text), anschaulich-bildlicher (Bild) sowie abstrakt-schematischer (Diagramm) Elemente präsentiert werden (vgl. ebd., S. 9). Ihre Diskontinuierlichkeit lässt sich damit erklären, dass sie durch eine spezifische, nicht lineare Kombination verschiedener Formen determiniert sind. Als solche werden sie zu einer Herausforderung, vor die ihre Rezipienten gestellt werden, weil „die Non-Linearität anders als bei den traditionellen, linearen Texten dazu [veranlasst] herauszufinden, was als zusammengehörig rezipiert wird“ (Żebrowska, 2014, S. 45; dazu auch Żebrowska, 2013). Infografiken als diskontinuerliche Darstellungsformen bilden ein sehr interessantes Objekt medienlinguistischer Forschung und evozieren Fragen nach Bedingungen und Folgen der semiotischen Ökonomie. Im Falle von hybriden Textformen, und Infografiken gelten als solche, lässt sich feststellen, dass die Tendenz dazu, Rezipienten möglichst schnell, zielgerichtet und effektiv anzusprechen, besonders stark ist. Martin Steinseifer zufolge lässt sich das damit erklären, dass Infografiken Informationen in einer solchen Weise präsentieren, in der relevante Zusammenhänge möglichst leicht zu erfassen sind (vgl. Steinseifer, 2013, S. 14). Wibke Weber und Hannes Rall, die sich mit der Frage der Hybridisierung interaktiver Infografiken beschäftigen, weisen auch darauf hin, dass sich diese Prozesse auf drei Ebenen vollziehen können (vgl. Weber \& Rall, 2015, S. 264 ff.). Die erste ist die formale Ebene, auf der sich die Vermischung der verschiedenen semiotischen Ressourcen als „äußerst komplexes multimodales Artefakt“ (ebd., S. 265) betrachten lässt. Die zweite Ebene, die die beiden Autorinnen als dramaturgisch-strukturelle klassifizieren, lässt die Frage danach stellen, wie eng die Dramaturgie einer Infografik mit deren Linearität bzw. Non-Linearität verknüpft ist. Je nach diesem Grad lassen sich Infografiken in drei Grundtypen unterteilen (vgl. ebd., S. 267). Der lineare Typ ist insofern von Vorteil, als er ,eine vom Produzenten(team) festgelegte Step-by-Step-Dramaturgie [vorgibt]“ (ebd.) und dadurch den Rezipienten durch die Infografik führt. Die Struktur der linearen Infografik ist sehr kohärent, was die Rezeption zwar erleichtert, allerdings bekommt der Rezipient häufig keinen Gesamtüberblick über alle angebotenen Inhalte, was natürlich als nachteilig anzusehen ist. Im Gegensatz dazu ist der nonlineare Typ dadurch gekennzeichnet, dass er einen individuellen Wahrnehmungsprozess ermöglicht. Interaktive Infografiken, die eine solche Struktur haben, müssen benutzerfreundlich gestaltet werden. Sonst besteht die Gefahr, dass nicht alle Daten und Fakten rezipiert werden. Der dritte, linear-nonlineare Typ gilt als „eine Mischung aus festgelegter Navigationsarchitektur seitens des Produzenten und einem gewissen Grad an Selektionsfreiheit seitens des Nutzers" (ebd., S. 270). Der Rezipient wird zwar durch die Infografik geführt, dies stört aber die Möglichkeit des individuellen Explorierens nicht. Die drei Typen sind vor allem unter interaktiven Infografiken zu 
finden. Aber auch unter Infografiken, von denen in den Printmedien Gebrauch gemacht wird und die nicht interaktiv sind, gibt es solche, die für Vertreter dieser Typen gehalten werden können.

Diskontinuierliche Darstellungsformen sind durch ihre modulare Struktur geprägt. Thomas Schröder zufolge „heißt Modularisierung, dass Textstruktur visualisiert wird und dass selektive Rezeption erleichtert wird“" (Schröder, 2010, S. 178). In so konstruierten Texten wird Verschiedenartiges vereint: Mitteilung mit Präsentation, Reflexion mit Anschauung, Verallgemeinerung mit Vereinzelung (vgl. Schmitz, 2003, S. 255). Wird die Frage nach dem Umgang mit solchen modularen Texten gestellt, dann wird klar, dass die Elemente ihrer Struktur nicht getrennt wirken, sondern als eine Gesamtbotschaft wahrgenommen und auf einmal holistisch erblickt werden. Weil sie eine gemeinsame Fläche teilen, werden sie auch als eine Gesamtbotschaft betrachtet, in der verschiedene Ressourcen zueinander in Beziehung stehen und synergetisch miteinander verflochten sind. Den Prozess ihrer Wahrnehmung nennt Ulrich Schmitz (2007) das Sehlesen und Flächen, die dem Prozess des Sehlesens unterliegen, bezeichnet er als Sehflächen: „Sehflächen sind Flächen, auf denen Texte und Bilder in geplantem Layout gemeinsame Bedeutungseinheiten bilden“ (Schmitz, 2011b, S. 3). Was im Falle von simultan und holistisch wahrgenommenen Gesamtbotschaften besonders vorteilhaft ist, ist ihre Fähigkeit, Aufmerksamkeit auf sich zu ziehen, größte Informationsmengen auf kleinstem Raum zu ermöglichen, vielfältige Präsentations-, Strukturierungs-, Orientierungs- und Rezeptionsweisen zu erlauben und den schnellen Blick zu unterstützen (vgl. ebd.). Die Bestandteile von Sehflächen, die im vorliegenden Beitrag und im Kontext der Medienmodularisierung bzw. -hybridisierung als sprachliche, bildliche oder diagrammatische Subflächen bezeichnet werden, werden als in irgendeiner Weise zusammengehörig verstanden. Infolge ihrer Zusammengehörigkeit wird die Bedeutung der Gesamtbotschaft durch wechselseitige Verbindung von kohäsiv wirkenden Subflächen potenziert. In Bezug auf die allgemeinen Regeln der Flächengestaltung und -wahrnehmung lassen sich am Beispiel der Infografik einige Mittel der Kohäsionsbildung angeben, die Martin Liebig (1999) als Gesetze formuliert und die die Konvention, die Richtung (von links nach rechts, von oben nach unten; das oberste Bildelement in der Mitte wird als Textanfang indiziert), die Nähe (nebeneinander platzierte Objekte werden als zugehörig wahrgenommen), die Einheitlichkeit (dieselbe Strichstärke, derselbe Farbton, dieselbe Farbintensität oder dieselbe Typografie) und die Dimension (Größenverhältnisse, Proportionen zwischen dominierenden und untergeordneten Elementen der Fläche) betreffen (vgl. Liebig, 1999, S. 115 ff.). Der Vorteil von diesen Regeln folgenden Sehflächen liegt darin, dass die auf diese Weise vermittelte Information gerade durch die Art und Weise ihrer Präsentation schneller und effektiver erfasst werden kann. Schmitz zufolge „,neigen [Texte] auf Sehflächen dazu, größere grammatisch-hierarchisch gebaute Bögen (z. B. komplexe Wortbildungen, längere Satzglieder oder ganze Sätze) zu vermeiden zugunsten jeweils kleinerer, in sich wenig strukturierter 
Einheiten“ (Schmitz, 2011b, S. 14). Das findet seine Bestätigung in Infografiken, in denen Diagramme und Bilder eine eigenständige Informationsfunktion übernehmen und somit als Alternative zum sprachlichen Text betrachtet werden können. „Infografiken können Text nicht nur ergänzen, sondern sogar ersetzen“, so Thomas Schröder (2010, S. 181). Die Infografiken, die das im vorliegenden Beitrag analysierte Korpus bilden, sind dadurch gekennzeichnet, dass sie journalistische, rein schriftsprachliche Texte nicht begleiten, sondern diese ersetzen und selbst als komplexe Darstellungsformen, als Sehflächen betrachtet werden.

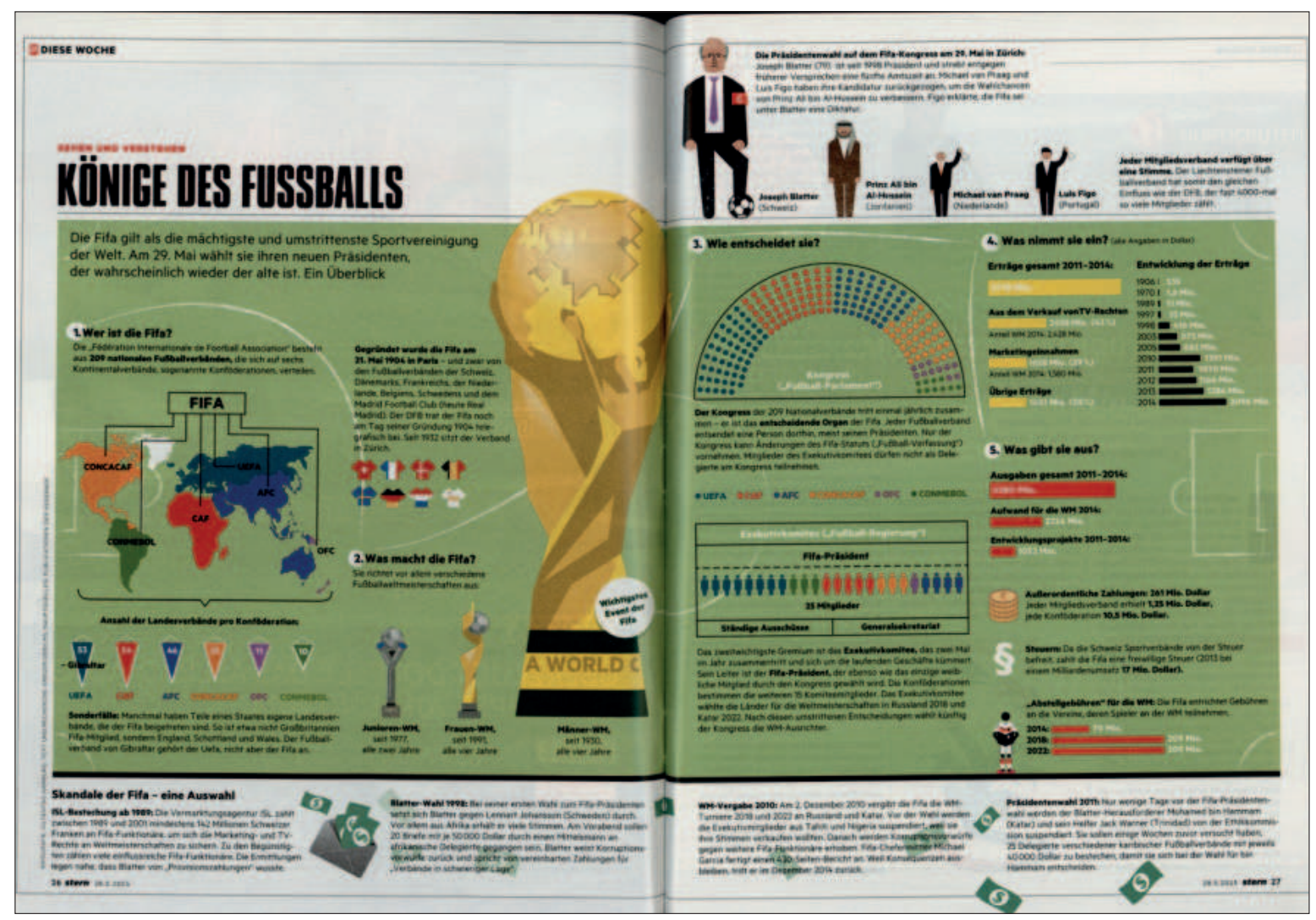

Abb. 1: Die FIFA-Sehfläche (Quelle: Stern, 23, 28.05.2015, 26-27)

Die in Abbildung 1 präsentierte Infografik begleitet keinen journalistischen Beitrag, sondern sie übernimmt seine Rolle, indem sie die wichtigsten Informationen über die FIFA-Organisation liefert. Diese werden in Form einer komplexen Sehfläche präsentiert, auf der sprachliche, bildliche und diagrammatische Ressourcen modular gebraucht werden. Von oben nach unten betrachtet besteht diese gesamte Sehfläche aus drei Modulen, von denen das mittlere am größten ist und die meisten Informationen auf einen Blick erfassbar macht. Daniela Wawra zufolge können einem Modul diejenigen Elemente einer Fläche zugeordnet werden, die dasselbe Design und den annähernd gleichen Umfang aufweisen (vgl. Wawra, 2007, S. 274). 


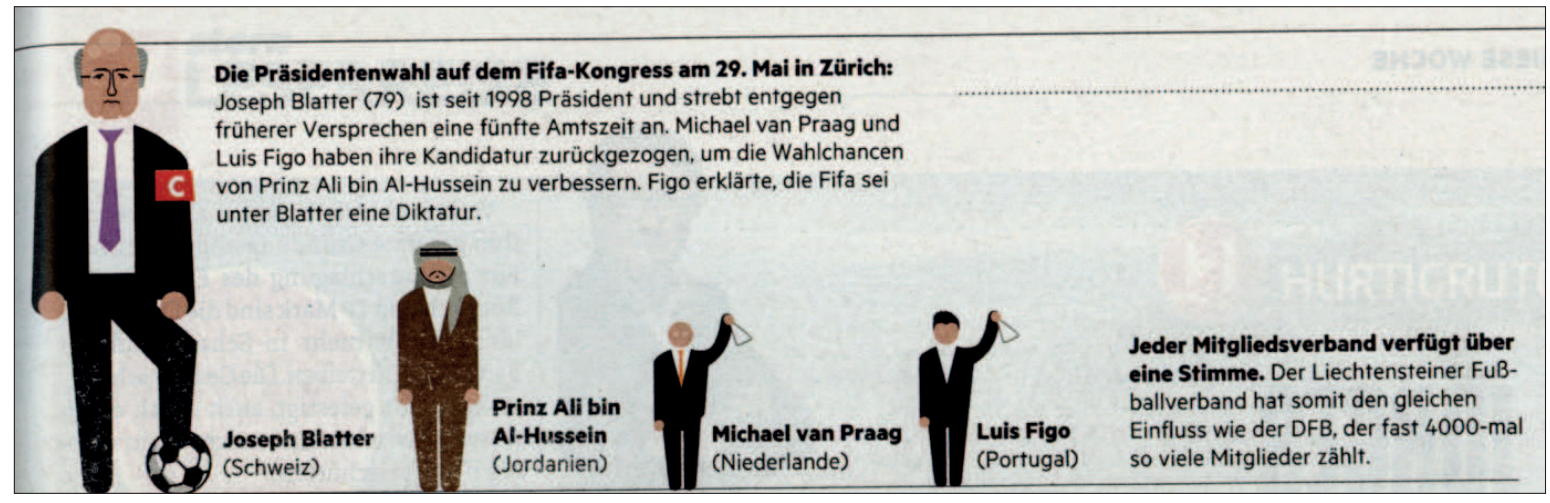

Abb. 2: Das obere Modul der FIFA-Sehfläche (Quelle: Stern, 23, 28.05.2015, 26-27)

Das erste Modul der FIFA-Infografik (Abb. 2) besteht aus sprachlichen und bildlichen Ressourcen. Mittels dieser Elemente wird die Frage der letzten Präsidentenwahl auf dem FIFA-Kongress am 29. Mai 2015 in Zürich thematisiert. Die Hintergründe des Kongresses werden sowohl beschrieben (sprachlich realisierte Mikrotexte zum Thema Stimmenverteilung) als auch bildlich dargestellt (vier Kandidaten zur Präsidentenwahl). Wenn angenommen wird, dass die bimodale, funktional-strukturelle Verknüpfung von Ressourcen für Sehflächen konstitutiv ist, lässt sich das obere Modul der FIFA-Infografik als Sehfläche im engeren Sinne klassifizieren. Besonders interessant ist sein bildlicher Teil, dem vier Männer-Figuren zugrunde liegen, die im Titel der Sehfläche als „Könige des Fussballs“ bezeichnet werden. Die größte Figur, die den Gewinner der Präsidentenwahl visualisiert, ist die von Joseph Blatter. Sein direkter Konkurrent, Prinz Ali bin Al Hussain, wird als zweiter präsentiert. Die zwei anderen Konkurrenten, Michael von Praag und Luis Figo, die ihre Kandidaturen zurückgezogen haben, werden mit weißen Tüchern winkend dargestellt. Im Kontext der Präsidentenwahl symbolisiert das in der Hand gehaltene weiße Tuch, dass der Kandidat sich zurückzieht und nicht mehr kämpfen will. Ohne den Text zu lesen, weiß man deshalb, dass nur zwei Kandidaten im Spiel geblieben sind. Joseph Blatter wird mit einem Fußball dargestellt, der als Symbol des Wahlerfolges zu interpretieren ist. Die sprachlichen und bildlichen Elemente dieser Sehfläche kontextualisieren sich wechselseitig, wodurch die Gesamtbedeutung dieser Botschaft potenziert wird.
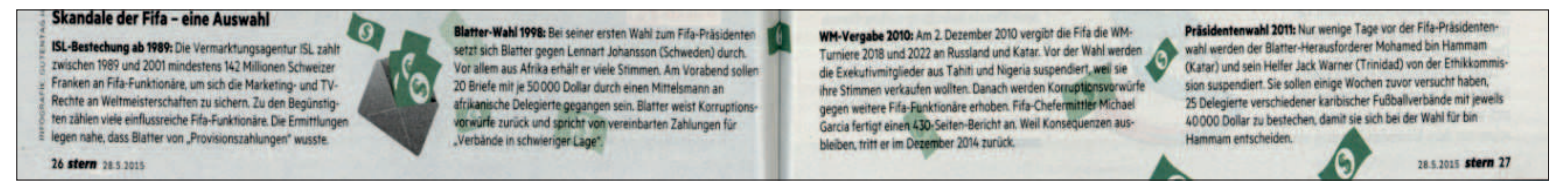

Abb. 3: Das untere Modul der FIFA-Sehfläche (Quelle: Stern, 23, 28.05.2015, 26-27)

Über eine ähnliche Struktur verfügt das in Abbildung 3 präsentierte untere Modul der FIFA-Infografik. Es ist auch bimodal und ist als Verflechtung sprachlicher und bildlicher Ressourcen anzusehen, die semantisch verbunden sind, weil sie an Skandale der 
FIFA anknüpfen. Im Vergleich zu den bildlichen Elementen des oberen Moduls (Abb. 2) haben die Bilder im unteren Modul (Abb. 3) eine geringere Anziehungskraft. Das lässt sich damit erklären, dass sie keine Personen, sondern Gegenstände (Briefumschläge mit dem US-Dollar als Symbol der Korruption in der Sport-Branche) darstellen. Sie wirken nicht so informativ wie z. B. das Bild einer Person, an dem man u. a. indirekt vermittelte Emotionen erkennen kann.

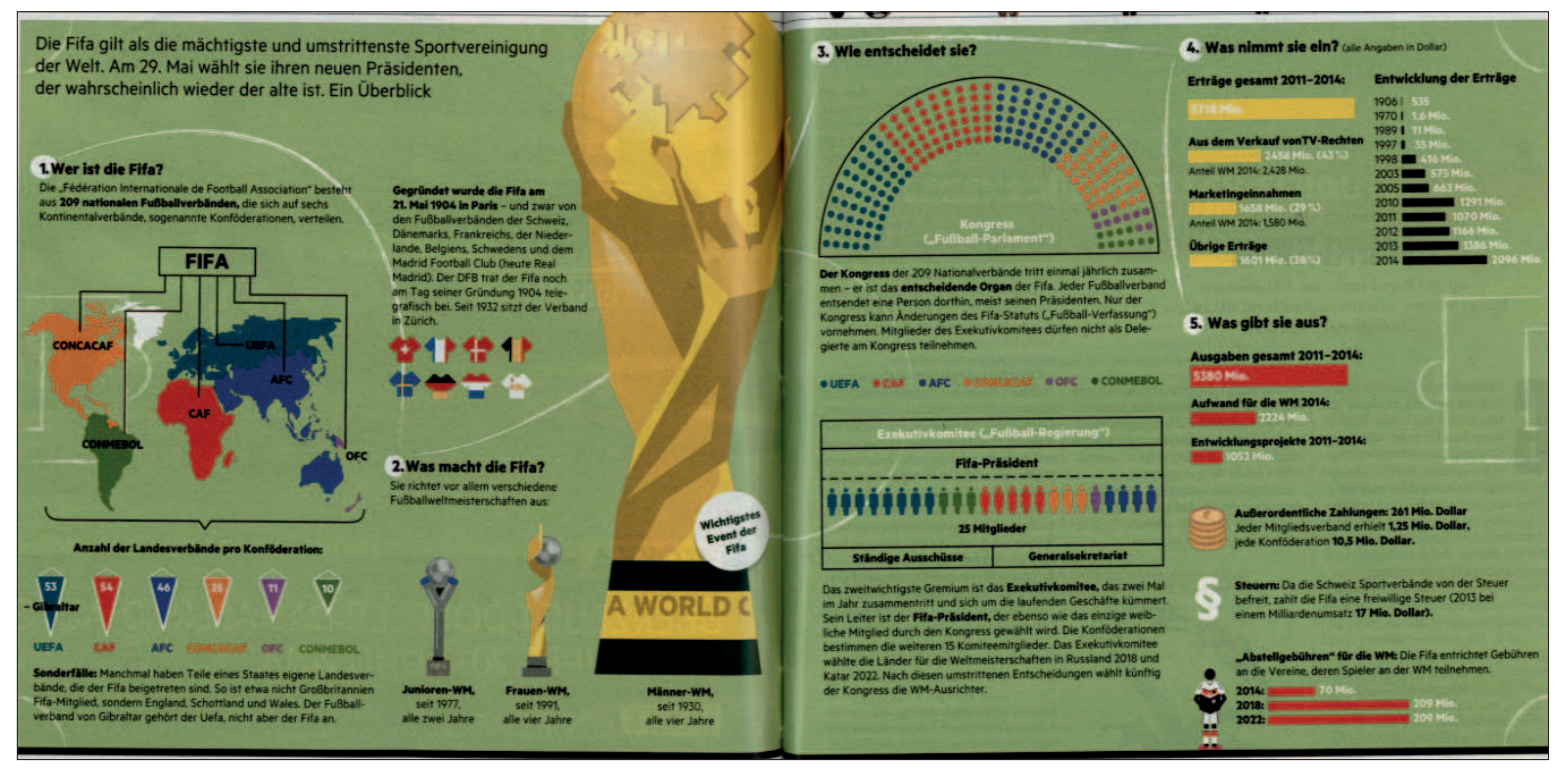

Abb. 4: Das mittlere Modul der FIFA-Sehfläche (Quelle: Stern, 23, 28.05.2015, 26-27)

Das mittlere Modul der analysierten FIFA-Infografik (Abb. 4) ist das umfangreichste und als solches liefert es den Rezipienten die meisten Informationen. Dies erfolgt auf dreierlei Weise: sprachlich, bildlich und diagrammatisch. Ein Kohäsionsmittel, dank dem all diese Ressourcen als Elemente einer Gesamtbotschaft betrachtet werden können, fungiert die grüne Farbe, die den Hintergrund des Moduls bildet und Assoziationen mit dem Fußballrasen evoziert. Wird diese Sehfläche nähert betrachtet, lässt sich feststellen, dass sie sich als linear-nonlineare Infografik klassifizieren lässt. Der Produzent macht von einer festgelegten Navigationsarchitektur Gebrauch, indem er mittels einer weißen Linie diese Sehfläche in fünf kleinere Teile (sog. Sehflächen im engeren Sinne) teilt und dadurch dem Rezipienten den Weg zur Infografik bahnt und die optimale Leserichtung suggeriert. Die erste der Sehflächen im engeren Sinne hat den Titel Wer ist die FIFA? und verfügt über drei Subflächen, die entsprechend aus sprachlichen (Sprache-Subfläche), bildlichen (zwei Bild-Subflächen: sechs Konföderationen/acht Gründer der FIFA) und diagrammatischen Elementen (Diagramm-Subfläche) bestehen. Die FIFA-Organisation wird somit sowohl beschrieben (Sprache-Subfläche), als auch doppelt visualisiert (Bild-Subfläche und Diagramm-Subfläche). Die zweite Sehfläche im engeren Sinne, die ausschließlich aus bildlichen und sparsam gebrauchten, sprachlichen Ressourcen besteht, gibt die Antwort auf die Frage, was die FIFA macht. Bemerkenswert ist, dass 
gerade ein Element dieser Sehfläche, und zwar die Visualisierung des goldenen FIFA-Pokals, das größte Element der gesamten FIFA-Sehfläche ist und über die größte Anziehungskraft verfügt. Die dritte Sehfläche im engeren Sinne hat den Titel Wie entscheidet sie? und ist bimodal. Die Rolle der FIFA-Gremien wird sowohl auf der Sprache-Subfläche thematisiert als auch in Form von den beiden Diagramm-Subflächen visualisiert. Die vierte Sehfläche im engeren Sinne wird mit Was nimmt sie ein? betitelt. Der Struktur nach unterscheidet sie sich von den anderen Sehflächen, weil sie sich nur zweier Diagramme bedient: Sprachliche Ressourcen werden dabei begrenzt gebraucht, bilden keine selbständige Sprache-Subfläche und dienen ausschließlich der informativen Unterstützung der beiden Diagramme. Die fünfte Sehfläche im engeren Sinne ist wiederum bimodal und fungiert als Kombination einer Diagramm- und einer Sprache-Subfläche. Unter allen analysierten Sehflächen im engeren Sinne, die das mittlere Modul der übergeordneten Sehfläche bilden, ist nur die Sehfläche 1 dadurch gekennzeichnet, dass ihre Struktur trimodal ist. Die Sehflächen 2, 3 und 5 sind bimodal und ausschließlich die Sehfläche 4 basiert auf diagrammatischen Ressourcen mit geringem sprachlichen Anteil. Die vom Produzenten gebrauchte weiße Linie, die dem Rezipienten den Weg zu den einzelnen Sehflächen bahnt und seinen Blick führt, ist hier ein Kohäsionsmittel und wird so gemalt, als ob sie die Bewegung eines Fußballs evozieren sollte. Dem Rezipienten wird somit vorgeschlagen, wie er sich mit dieser Sehfläche bekannt machen kann. Dadurch kommt ein gewisser Grad an Selektionsfreiheit seitens des Nutzers zum Ausdruck: Der Rezipient wird zwar durch die Infografik geführt, aber das stört die Möglichkeit des individuellen Explorierens nicht.

Andere Möglichkeiten und Funktionen des Infografischen werden in der in Abbildung 5 präsentierten Infografik genutzt, die aufgrund ihrer Ähnlichkeit mit einer Karte für eine Art Kartogramm gehalten werden kann. Einerseits wird damit ihre terminologische Nähe zu anderen Diagrammtypen ausgedrückt, andererseits handelt es sich dabei um einen Kartentyp relativ einfacher Bauart, dessen Aufgabe darin besteht, dem Rezipienten topografische Informationen zu liefern. Diese Art der im Journalismus immer häufiger gebrauchten Infografik ist einerseits durch ihre lagegetreue Abbildung und andererseits durch die Kategorisierung (als Stadt, Land, Bau) anhand der festgelegten Elemente gekennzeichnet. In Bezug auf die neuere Kartosemiotik weist Thomas Lischeid $(2009,2012)$ darauf hin, dass sich kartografische Elemente zugleich sowohl auf der Wort- als auch auf der Satz-Ebene bewegen. Bei der Wort-Ebene sind das ihre verbalen Elemente, wie z. B. einzelne Städte- oder Schlossnamen (im analysierten Fall geht es um das Berliner Stadtschloss) und bei der Satz-Ebene handelt es sich darum, dass jedes Kartenzeichen satzförmig verbalisierbar ist (das Objekt $\mathrm{x}$ liegt an der Stelle y) (vgl. Lischeid, 2012, S. 331). Die Hauptfunktion kartografisch orientierter Infografiken besteht darin, Informationen über topografische Verhältnisse zu liefern und die Orientierung im Raum zu erleichtern. Dies erfolgt u. a. dadurch, dass diese Darstellungen ein 3D-Format intendieren. Dadurch entsteht der spezifische Eindruck einer räumlichen Darstellung. 


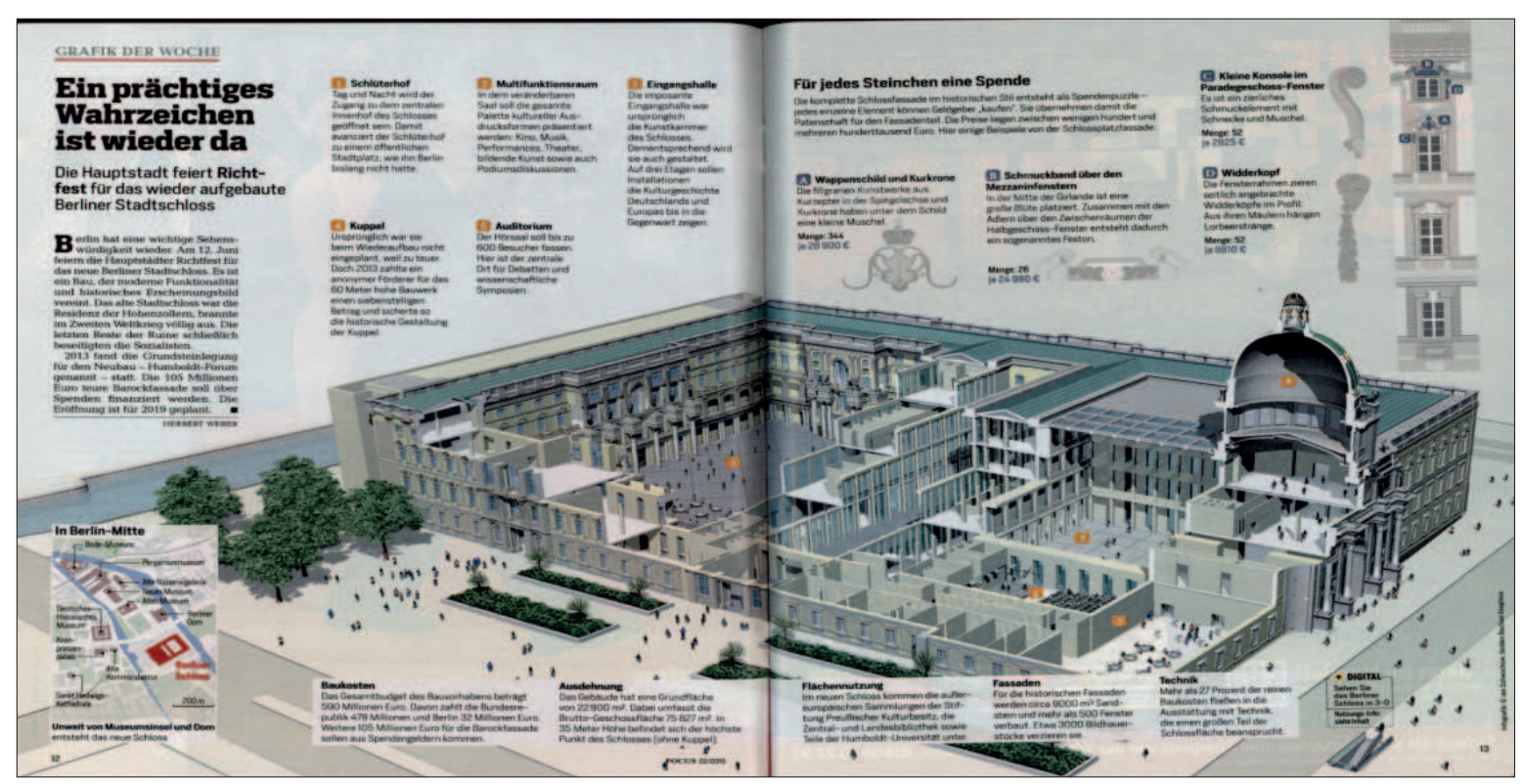

Abb. 5: Die Schloss-Sehfläche (Quelle: Focus, 12/15, 23.05.2015, 12-13)

Die in Abbildung 5 präsentierten Seiten aus dem Focus bilden somit eine bimodale Sehfläche, die von sprachlichen und bildlichen Ressourcen Gebrauch macht, um das neue Image des Berliner Stadtschlosses zu präsentieren. Auf dieser Sehfläche ist zwar kein Diagramm zu sehen, aber die Art und Weise, wie das Schloss präsentiert wird, gibt dieser Sehfläche einen infografischen Charakter. Ulrich Schmitz vertritt die Meinung, dass jede Sehfläche gewissermaßen ein Diagramm ist: „Seine Bestandteile sind per Design so aufeinander bezogen, dass der Nutzer die gemeinten Beziehungen seinen Zielen entsprechend auswählen, erkennen, nachvollziehen und praktisch nutzen kann"(Schmitz, 2011a, S. 102). Die Schloss-Sehfläche besteht aus zwei Subflächen: Die Bild-Subfläche stellt das Berliner Stadtschloss dar, während die Legende die Sprache-Subfläche bildet, die alles thematisiert, was auf der Bild-Subfläche zu sehen ist. Die Schloss-Sehfläche wird vertikal strukturiert: Die Sprache-Subfläche befindet sich über der Bild-Subfläche, aber gerade die Bild-Subfläche ist hier der Einstieg in diese Sehfläche, weil das dabei präsentierte Schlossgebäude zum größten visuellen Element der gesamten Sehfläche wird. Das Schloss wird dabei so detailliert visualisiert, dass sowohl seine innere Architektur als auch die daraus resultierenden Funktionen dem Auge des Rezipienten gut zugänglich sind und problemlos verstanden werden können. „Dabei wird eine breite Skala zwischen Ergonomie und Ästhetik bespielt“", so Schmitz (2011a, S. 86).

Aus der Analyse der in Abb. 1 und 5 dargestellten Infografiken geht hervor, dass sie zwei verschiedene Typen von Sehflächen repräsentieren. Je nachdem, welcher Art der Modularisierung eine Sehfläche unterliegt, werden Sehflächen in zwei Gruppen geteilt. Sehflächen im engeren Sinne sind dadurch gekennzeichnet, dass sie mindestens bimodal sind, in einem Kognitionsakt holistisch wahrgenommen werden und semantisch zusammenhängen. In diesem Sinne sind das obere und das untere Modul der FIFA-Infografik 
sowie die Schloss-Infografik Sehflächen im engeren Sinne zuzuordnen. Das Entscheidungskriterium ist ihre bimodale Struktur (sie bestehen ausschließlich aus sprachlichen und bildlichen Ressourcen). Wird danach gefragt, wodurch der infografische Charakter dieser Sehflächen zum Ausdruck kommt, dann muss darauf hingewiesen werden, dass z. B. die Bild-Subfläche des oberen Moduls (FIFA-Sehfläche), auf der vier Figuren unterschiedlicher Größe präsentiert werden, Assoziationen mit einem Diagramm evoziert. Die Schloss-Sehfläche verfügt zwar über keine Diagramm-Subfläche, aber sie stellt das Berliner Stadtschloss als ein 3D-Format dar, wodurch die Vorstellung eines Kartogramms evoziert wird und die Sehfläche wie eine Infografik wirkt. Wird danach gefragt, welche Kriterien für Sehflächen im weiteren Sinne konstitutiv sind, kann am Beispiel der FIFA-Sehfläche (das mittlere Modul) beobachtet werden, dass dieses Modul durch fünf kleinere Module, d. h. Sehflächen im engeren Sinne, gebildet wird. Deshalb kann dieses als Sehfläche im weiteren Sinne angesehen werden, die sich weiter in mehrere kleinformatige Sehflächen teilen lässt. Das beweist, dass Sehflächen im weiteren Sinne über eine tiefere Struktur verfügen und eine weitere Gliederung erlauben.

\section{Fazit}

Zusammenfassend lässt sich feststellen, dass sich sprachliche, bildliche und diagrammatische Elemente in Infografiken die Arbeit der Informationsvermittlung teilen. Je stärker die visuelle Gestaltung zur Herstellung der Gesamtbedeutung beiträgt, desto mehr Aufgaben gibt Grammatik an andere Ordnungsinstanzen ab. Journalistische Infografiken, die als Vertreter der visuellen Publizistik in dem vorliegenden Beitrag im Zentrum des Forschungsinteresses stehen, zeigen, dass die Arbeitsteilung, zu der es zwischen sprachlichen und grafischen Ressourcen kommt, nicht nur im Bereich der Werbung festzustellen ist, sondern alle Sehflächen betrifft, auf denen visuelles Design als oberste semiotische Gestaltungsinstanz wirkt. Das Design, das bei Infografiken enorm wichtig ist und als eine Art Integrator und Moderator anzusehen ist, verbindet die einzelnen Modi zu einem sinnvollen Ganzen, akzentuiert Inhalte, weist den Rezipienten an, leitet ihn und gibt ihm Orientierung. Somit gelten Infografiken als Blickfang und Mitteilung, die dem Rezipienten attraktive und ergonomische Informationsvermittlung garantieren. Das multimodale Zusammenspiel, das sich in den analysierten Infografiken beobachten lässt, ist als designerische Tätigkeit zu verstehen, die mittels verschiedener Zeichenressourcen Inhalte zum Ausdruck bringt. Dabei werden die Infografiken zu semiotischen Artefakten, die durch ihre mediale Manifestation Verbreitung finden. Während Design, verstanden als intentionale Gestaltung von Oberfläche, die kommunikative Mittlerrolle zwischen Inhalt und Ausdruck spielt, dient das Verbreitungsmedium als Vermittler zwischen Produktion und Rezeption. Das bedeutet, dass mit dem Trägermedium weitere Modifikationen in der Textsorte Infografik eintreten können. 


\section{Literatur}

Berger, A.A. (1989). Seeing is Believing. An Introduction to Visual Communication. Mountain View: Mayfield Publishing Company.

Blum, J. \& Bucher, H.-J. (1998). Die Zeitung: Ein Multimedium: Textdesign - ein Gestaltungskonzept für Text, Bild und Grafik. Sonderheft „Sage/Schreibe“. Konstanz: UVK.

Bouchon, C. (2007). Infografiken: Einsatz, Gestaltung und Informationsvermittlung. Boizenburg: Hülsbusch.

Gudehus, J. (2010). Das Lexikon der visuellen Kommunikation. Mainz: Hermann Schmidt.

Hauser, S. \& Luginbühl, M. (2015). Hybridisierung und Ausdifferenzierung - Einführende begriffliche und theoretische Anmerkungen. In S. Hauser \& M. Luginbühl (Hrsg.), Hybridisierung und Ausdifferenzierung. Kontrastive Perspektiven linguistischer Medienanalyse (S. 7-30). Bern-Berlin-New York: Peter Lang.

Hermann, U. (1982). Knaurs Herkunftswörterbuch, Etymologie und Geschichte von 10.000 interessanten Wörtern. Berlin: Deutscher Bücherbund.

Knieper, T. (1995). Infographiken: das visuelle Informationspotenzial der Tageszeitung. München: Reinhard Fischer.

Küpper, N. (2010). Journalisten-Werkstatt: Infografik. Sonderheft von Der Österreichische Journalist, medium magazin und Schweizer Journalist. Salzburg: Oberauer.

Liebig, M. (1999). Die Infografik. Konstanz: UVK.

Lischeid, T. (2009). Text-Bild-Diagramm. Ein semiotisches Modell diskontinuierlicher Darstellungsformen am Beispiel der Infografik. In N. Hinrichs \& A. Limburg (Hrsg.), Gedankenstriche - Reflexionen über Sprache als Ressource. Beiträge zur Didaktik an der Schnittstelle Schule/Hochschule. Festschrift für Prof. Dr. Wolfgang Boettcher zum 65. Geburtstag (S. 72-91). Tübingen: Stauffenburg.

Lischeid, T. (2012). Diagrammatik und Mediensymbolik. Multimodale Darstellungsformen am Beispiel der Infografik. Duisburg: UVRR.

Schmitz, U. (2003). Deutsche Schriftsprache in hypermedialer Umgebung. Zeitschrift für germanistische Linguistik, 31 (2), 253-273.

Schmitz, U. (2007). Sehlesen. Text-Bild-Gestalten in massenmedialer Kommunikation. In K.S. Roth \& J. Spitzmüller (Hrsg.), Textdesign und Textwirkung in der massenmedialen Kommunikation (S. 93108). Konstanz: UVK.

Schmitz, U. (2011a). Blickfang und Mitteilung. Zur Arbeitsteilung von Design und Grammatik in der Werbekommunikation. Zeitschrift für angewandte Linguistik, 54, 79-109.

Schröder, T. (2010). Die Bilder-Zeitung: wie ein Textmedium zu einem Medium der visuellen Kommunikation wird. In H.-J. Bucher \& T. Gloning \& K. Lehnen (Hrsg.), Neue Medien - Neue Formate. Ausdifferenzierung und Konvergenz in der Medienkommunikation (S. 169-188). Frankfurt am MainNew York: Campus Verlag.

Steinseifer, M. (2013). Texte sehen - Diagrammatologische Impulse für die Textlinguistik. Zeitschrift für Germanistische Linguistik, 41 (1), 8-39.

Stöckl, H. (2012). Finanzen verbalisieren - Die Text-Bild-Sorte Infographik. OBST Osnabrücker Beiträge zur Sprachtheorie, 81, 177-199.

Stöckl, H. (2015). Bewegung auf der Titelseite - Ausdifferenzierung und Hybridisierung durch Sprache-Bild-Texte. In H.-J. Bucher \& T. Gloning \& K. Lehnen (Hrsg.), Neue Medien - Neue Formate. Ausdifferenzierung und Konvergenz in der Medienkommunikation (S. 235-259). Bern: Peter Lang.

Wawra, D. (2007). Beziehungsgestaltung und `Image-Building`. In K.S. Roth \& J. Spitzmüller (Hrsg.), Textdesign und Textwirkung in der massenmedialen Kommunikation (S. 267-289). Konstanz: UVK.

Weber, W. \& Rall, H. (2015). Hybride Konstruktionen bei interaktiven Infografiken. In S. Hauser \& M. Luginbühl (Hrsg.), Hybridisierung und Ausdifferenzierung. Kontrastive Perspektiven linguistischer Medienanalyse (S. 261-288). Bern-Berlin-New York: Peter Lang. 
Żebrowska, E. (2013). Text-Bild-Hypertext. Frankfurt am Main-Berlin-Bern-Bruxelles-New York-Oxford-Wien: Peter Lang AG.

Żebrowska, E. (2014). Visuelle Texte. In G. Antos \& R. Opiłowski \& J. Jarosz (Hrsg.), Sprache und Bild im massenmedialen Text. Formen, Funktionen und Perspektiven im deutschen und polnischen Kommunikationsraum (S. 43-54). Wrocław-Dresden: ATUT-Neisse Verlag.

\section{Internetquellen}

Schmitz, U. (2011b). Sehflächenforschung. Eine Einführung. Abgerufen von www.linse.uni-due.de [Zugriff: 1.02 .2014$]$

Stöckl, H. (2010). Sprache-Bild-Texte lesen. Bausteine zur Methodik einer Grundkompetenz. Abgerufen von http://stoeckl.sbg.ac.at/Stoeckl/Publikationen_2_files/Stoeckl_\%20Sprache-Bild-Texte_2010. pdf [Zugriff: 10.11.2015] 